\title{
The Transmuted Weibull Lomax Distribution: Properties and Application
}

\author{
Ahmed Z. Afify \\ Department of Statistics, Mathematics and Insurance \\ Benha University, Egypt \\ ahmed.afify@fcom.bu.edu.eg \\ Zohdy M. Nofal \\ Department of Statistics, Mathematics and Insurance \\ Benha University, Egypt \\ dr.zohdynofal@fcom.bu.edu.eg \\ Haitham M. Yousof \\ Department of Statistics, Mathematics and Insurance \\ Benha University, Egypt \\ haitham.yousof@fcom.bu.edu.eg \\ Yehia M. El Gebaly \\ Department of Statistics, Mathematics and Insurance \\ Benha University, Egypt \\ Yehia1958@yahoo.com \\ Nadeem Shafique Butt \\ Department of Family and Community Medicine, Rabigh \\ King Abdul Aziz University, Jeddah, Kingdom of Saudi Arabia \\ nshafique@kau.edu.sa
}

\begin{abstract}
A new five parameter model is proposed and stutied. The new distribution generalizes the Weibull Lomax distribution introduced by Tahir et al. (2015) and is referred to as transmuted Weibull Lomax (TWL) distribution. Various structural properties of the new model including ordinary and incomplete moments, quantiles, generating function, probability weighted moments, Rényi and q-entropies and order statistics are derived. We proposed the method of maximum likelihood for estimating the model parameters. The usefulness of the new model is illustrated through an application to a real data set.
\end{abstract}

Keywords: Weibull Lomax, Probability Weighted Moments, Entropy, Order Statistics, Maximum Likelihood.

\section{Introduction}

In fact, there are hundreds of continuous univariate distributions. However, in recent years, applications from the environmental, financial, biomedical sciences, engineering among others, have further shown that data sets following the classical distributions are more often the exception rather than the reality. Since there is a clear need for extended forms of these distributions a significant progress has been made toward the generalization of some well-known distributions and their successful application to 
problems in areas such as engineering, finance, economics and biomedical sciences, among others.

This paper aims to introduce a new generalization to the Lomax distribution using the transmutation map approach introduced by Shaw and Buckley (2007). The new model which generalizes the Weibull Lomax (WL) distribution introduced by Tahir et al. (2015) is referred to as the transmuted Weibull Lomax (TWL) distribution.

The Lomax (or Pareto II) distribution has wide applications in many fields such as income and wealth inequality, medical and biological sciences, engineering, size of cities actuarial science, lifetime and reliability modeling. In the lifetime context, the Lomax model belongs to the family of decreasing failure rate see Chahkandi and Ganjali (2009) and arises as a limiting distribution of residual lifetimes at great age see Balkema and de Hann (1974). For more information about the Lomax distribution and Pareto family are given in Arnold (1983) and Johnson et al. (1994).

Many authors constructed generalizations of the Lomax distribution. For example, Abdul-Moniem and Abdel-Hameed (2012) studied exponentiated Lomax (EL), Ghitany et al. (2007) introduced Marshall-Olkin extended Lomax (MOEL), Lemonte and Cordeiro (2013) investigated beta Lomax (BL), Kumaraswamy Lomax (KwL) and McDonald Lomax (McL) and Cordeiro et al. (2013) introduced gamma Lomax (GL) distributions. Recently Tahir et al. (2015) introduced the Weibull Lomax (WL) distribution and studied its mathematical and statistical properties.

The cumulative distribution function $(c d f)$ (for $x>0$ ) of the Weibull lomax distribution is given by

$$
G(x, a, b, \alpha, \beta)=1-\exp \left\{-a\left\{\left[\left(1+\frac{x}{\beta}\right)^{\alpha}-1\right]\right\}^{b}\right\}
$$

where $\beta$ is a scale parameter, $\alpha, a$ and $b$ are shape parameters. The corresponding probability density function $(p d f)$ is given by

$$
\begin{gathered}
g(x, a, b, \alpha, \beta)=\frac{a b \alpha}{\beta}\left(1+\frac{x}{\beta}\right)^{b \alpha-1}\left[1-\left(1+\frac{x}{\beta}\right)^{-\alpha}\right]^{b-1} \\
\exp \left\{-a\left\{\left[\left(1+\frac{x}{\beta}\right)^{\alpha}-1\right]\right\}\right.
\end{gathered}
$$

The aim of this paper is to provide more flixible extension of the Weibull Lomax (WL) distribution using the transmutation map technique introduced by Shaw and Buckley (2007) called transmuted Weibull Lomax (TWL) distribution. 
According to the Quadratic Rank Transmutation Map, (QRTM), approach a random variable $X$ is said to have transmuted distribution if its cumulative distribution function $(c d f)$ is given by

$$
F(x)=(1+\lambda) G(x)-\lambda G^{2}(x),
$$

where $G(x)$ is the $(c d f)$ of the base distribution, which on differentiation yields

$$
f(x)=g(x)[1+\lambda-2 \lambda G(x)],|\lambda| \leq 1,
$$

where $f(x)$ and $g(x)$ are the corresponding $p d f s$ associated with $c d f s F(x)$ and $G(x)$ respectively. For more information about the quadratic rank transmutation map is given in Shaw and Buckley (2007). Observe that at $\lambda=0$, we have the base distribution.

Recently, various generalizations have been introduced based on the transmutation map approach. Afify et al. (2014) introduced the transmuted complementary Weibull geometric distribution and studied its mathematical. Ashour and Eltehiwy (2013) introduced the transmuted Lomax distribution.

In this paper we provide mathematical and statistical properties of the exponentiated Weibull Lomax (TWL) distribution. The rest of the paper is outlined as follows. In Section 2, we define the subject distribution and provide the graphical presentation for its $p d f$ and $h r f$. In Section 3, we provide a very useful expansions for the $p d f$ and $c d f$ of the new model. Section 4 provides statistical properties including quantile functions, random number generation, ordinary and incomplete moments, moment generating functions, mean deviations, probability weighted moments and Rényi entropy are derived. In Section 5, the order statistics and its moments are discussed. The maximum likelihood estimates (MLEs) and the asymptotic confidence intervals of the unknown parameters are demonstrated in Section 6. In section 7, the TWL distribution is applied to a real data set to illustrate its usefulness. Finally, some concluding remarks are given in section 8 .

\section{The TWL Distribution}

The Transmuted Weibull Lomax (TWL) distribution and its sub-models are presented in this section. The random variable $(r . v) X$ is said to have an TWL distribution, denoted by $X: T W L(x, \alpha, \beta, \lambda, a, b)$, if its $c d f$ is given by 


$$
\begin{gathered}
F(x, \alpha, \beta, \lambda, a, b)=\left(1-\exp \left\{-a\left\{\left(1+\frac{x}{\beta}\right)^{\alpha}-1\right\}\right\}\right) \\
\quad \times\left\{(1+\lambda)-\lambda\left(1-\exp \left[-a\left\{\left(1+\frac{x}{\beta}\right)^{\alpha}-1\right\}^{b}\right]\right)\right\} .
\end{gathered}
$$

The corresponding $p d f$ of $X$ is given by

$$
\begin{aligned}
& f(x, \alpha, \beta, \lambda, a, b)=\frac{a b \alpha}{\beta}\left(1+\frac{x}{\beta}\right)^{b \alpha-1}\left[1-\left(1+\frac{x}{\beta}\right)^{-\alpha}\right]^{b-1} \exp \left\{-a\left\{\left(1+\frac{x}{\beta}\right)^{\alpha}-1\right\}^{b}\right\} \\
& \quad \times\left\{(1+\lambda)-2 \lambda\left(1-\exp \left[-a\left\{\left(1+\frac{x}{\beta}\right)^{\alpha}-1\right\}\right)\right\} .\right.
\end{aligned}
$$

where $\beta$ is a scale parameter representing the characteristic life, $\alpha$, and $\eta$ are shape parameters representing the different patterns of the TWL distribution and $\lambda$ is the transmuted parameter. The reliability function (rf), and cumulative hazard rate function (chrf) of the r.v $X$ are given by

$$
R(x, \alpha, \beta, \lambda, a, b)=1-\left\{\begin{array}{c}
\left(1-\exp \left[-a\left\{\left(1+\frac{x}{\beta}\right)^{\alpha}-1\right\}^{b}\right]\right) \\
\left.(1+\lambda)-\lambda\left(1-\exp \left[-a\left\{\left(1+\frac{x}{\beta}\right)^{\alpha}-1\right\}\right]\right)\right\},
\end{array}\right\},
$$

and

$$
H(x, \alpha, \beta, \lambda, a, b)=-\ln \left\{1-\left\{1-\exp \left[-a\left\{\left(1+\frac{x}{\beta}\right)^{\alpha}-1\right\}^{b}\right]\right)\right\} .
$$

respectively. 
Figure 1 (a), (b), (c) and (d) provide some plots of the TWL density curves for different values of the parameters $\alpha, \beta, \lambda, a$ and $b$. Plots of the hazard rate function of TWL for selected parameter values are given in Figure 2.

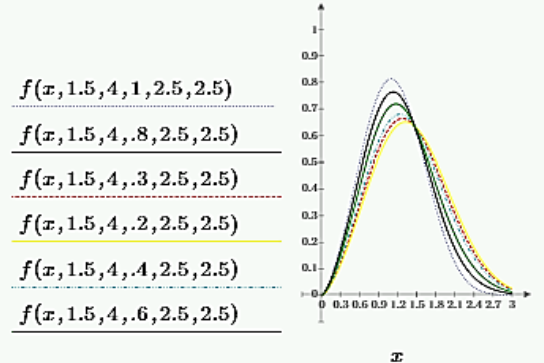

(a)

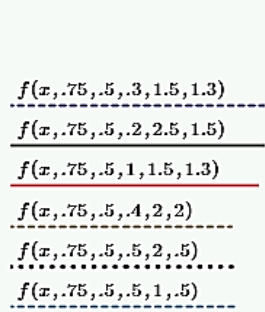

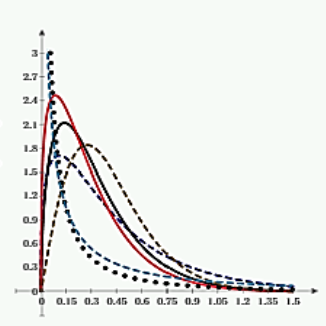

(c)
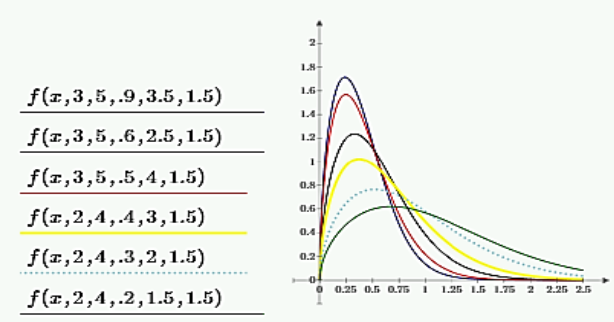

(b)

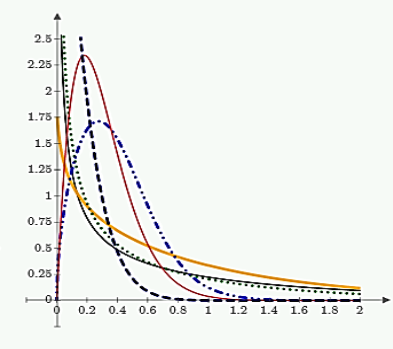

(d)

Figure 1: Plots of the TWL density function for some parameter values. (a) For different values of $\lambda, a$ and $b$ with $\alpha=1.5$ and $\beta=4$. (b) For different values of $\lambda, a$ and $b$ with $\alpha=2,3$ and $\beta=4,5$. (c) For different values of $\lambda, a$ and $b$ with $\alpha=0.75$ and $\beta=0.5$. (d) For different values of $\lambda, a$ and $b$ with $\alpha=2$ and $\beta=1$.
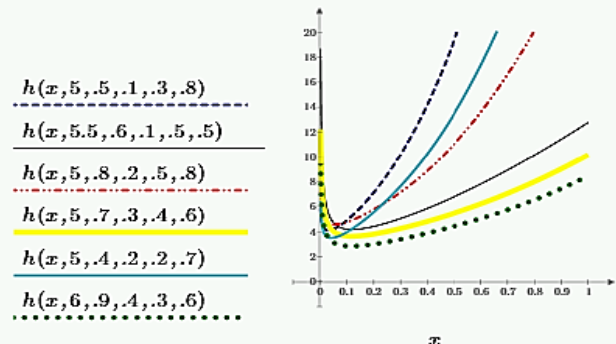

(a)

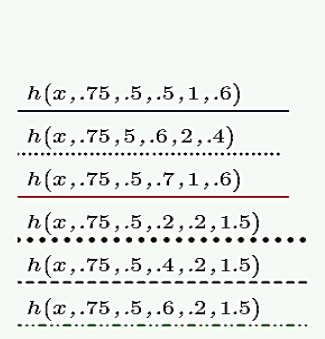

$h(x, .75, .5, .6, .2,1.5)$

(c)

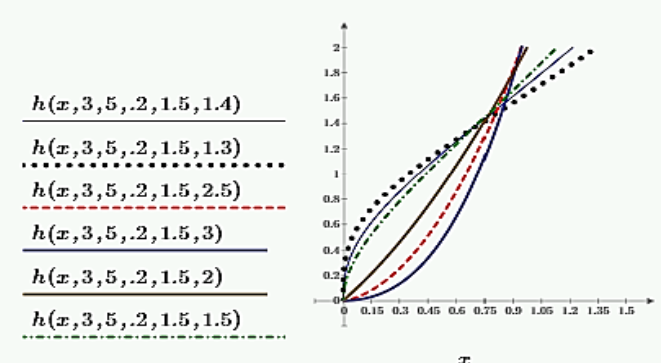

(b)

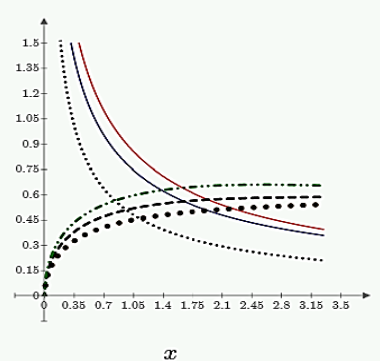

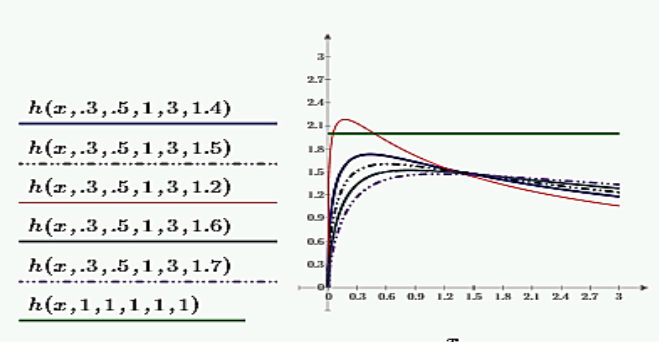

(d)

Figure 2: Plots of the TWL hazard rate function for some parameter values 


\section{Mixture Representation}

The TWL density function given in (4) can be rewritten as

$$
\begin{aligned}
& f(x, \alpha, \beta, \lambda, a, b)=\operatorname{abg}(x) \frac{G(x)^{b-1}}{\bar{G}(x)^{b+1}} \sum_{i, l=0}^{\infty}(1+\lambda)^{i+1}(2 \lambda)^{i}(-1)^{i+l} \\
& \quad \times\left(\begin{array}{l}
1 \\
i
\end{array}\right)\left(\begin{array}{l}
i \\
l
\end{array}\right) \exp \left\{-a(l+1)\left(\frac{G(x)}{\bar{G}(x)}\right)^{b}\right\} .
\end{aligned}
$$

By inserting (1) and (2) in Equation (5), we obtain

$$
\begin{aligned}
& f(x, \alpha, \beta, \lambda, a, b)=\frac{a b \alpha \gamma}{\beta}\left(1+\frac{x}{\beta}\right)^{-(\alpha+1)} \frac{\left[1-\left(1+\frac{x}{\beta}\right)^{-\alpha}\right]^{b-1}}{\left\{1-\left[1-\left(1+\frac{x}{\beta}\right)^{-\alpha}\right]\right\}^{b+1}} \\
& \quad \times \sum_{i, l=0}^{\infty}(1+\lambda)^{i+1}(2 \lambda)^{i}(-1)^{i+l}\left(\begin{array}{l}
1 \\
i
\end{array}\right)\left(\begin{array}{l}
i \\
l
\end{array}\right) \\
& \quad \times \exp \left[-a(l+1)\left(\frac{\left[1-\left(1+\frac{x}{\beta}\right)^{-\alpha}\right]}{1-\left[1-\left(1+\frac{x}{\beta}\right)^{-\alpha}\right]}\right)^{b}\right] .
\end{aligned}
$$

Equation (6) can be expanded in power series as

Let

$$
L=\exp \left[-a(i+1)\left(\frac{\left[1-\left(1+\frac{x}{\beta}\right)^{-\alpha}\right]}{1-\left[1-\left(1+\frac{x}{\beta}\right)^{-\alpha}\right]}\right] .\right.
$$

By expanding the exponential function in $L$, we obtain

$$
L=\sum_{k=0}^{\infty} \frac{(-1)^{k} a^{k}(l+1)^{k}}{k !} \frac{\left[1-\left(1+\frac{x}{\beta}\right)^{-\alpha}\right]^{k b}}{\left\{1-\left[1-\left(1+\frac{x}{\beta}\right)^{-\alpha}\right]\right\}^{k b}} .
$$


Inserting this expansion in Equation (6) and, after some simplification, we obtain

$$
\begin{aligned}
& f(x, \alpha, \beta, \lambda, a, b)=\frac{b \alpha}{\beta} \sum_{i, k, l=0}^{\infty} \frac{(-1)^{i+k+l} a^{k+1}(l+1)^{k}(1+\lambda)^{i+1}(2 \lambda)^{i}}{k !}\left(\begin{array}{l}
1 \\
i
\end{array}\right)\left(\begin{array}{l}
i \\
l
\end{array}\right)\left(1+\frac{x}{\beta}\right)^{-(\alpha+1)} \\
& \times\left[1-\left(1+\frac{x}{\beta}\right)^{-\alpha}\right]^{k b+b-1}\left\{1-\left[1-\left(1+\frac{x}{\beta}\right)^{-\alpha}\right]\right\}^{-k b-b+1} .
\end{aligned}
$$

Appluing a power series expansion again, we get

$$
\begin{aligned}
& f(x, \alpha, \beta, \lambda, a, b)=\sum_{j, i, k, l=0}^{\infty} \frac{(-1)^{j+i+k+l} a^{k+1}(l+1)^{k}(1+\lambda)^{i+1}(2 \lambda)^{i}}{j ! k !} \\
& \times\left(\begin{array}{l}
1 \\
i
\end{array}\right)\left(\begin{array}{l}
i \\
l
\end{array}\right) \frac{b}{[(k+1) b+j]} \frac{\Gamma[(k+1) b+j+1]}{\Gamma[(k+1) b+1]} \\
& \quad \times \frac{\alpha}{\beta}[(k+1) b+j]\left(1+\frac{x}{\beta}\right)^{-(\alpha+1)}\left\{1-\left(1+\frac{x}{\beta}\right)^{-\alpha}\right\}^{[(k+1) b+j]-1}
\end{aligned}
$$

Equation (7) can be rewritten as a mixture of exponentiated Lomax (EL) densities

$$
f(x, \alpha, \beta, \gamma, a, b)=\sum_{j, i, k, l=0}^{\infty} u_{j, i, k, l} h_{a, \alpha,(k+1) b+j}(x),
$$

where

$$
\begin{aligned}
& u_{j, i, k, l}=\frac{(-1)^{j+i+k+l} a^{k+1}(l+1)^{k}(1+\lambda)^{i+1}(2 \lambda)^{i}}{j ! k !} \\
& \times\left(\begin{array}{l}
1 \\
i
\end{array}\right)\left(\begin{array}{l}
i \\
l
\end{array}\right) \frac{b}{[(k+1) b+j]} \frac{\Gamma[(k+1) b+j+1]}{\Gamma[(k+1) b+1]} .
\end{aligned}
$$

and $h_{a, \alpha,(k+1) b+j}(x)$ is the EL density with parameters $a, \alpha$ and $(k+1) b+j$. Therefore, the properties TWL distribution can be derived form those of the EL distribution.

The $c d f$ of the TWL in (3) can be expressed in the mixture form

$$
F(x, \alpha, \beta, \lambda, a, b)=\sum_{j, i, k, l=0}^{\infty} u_{j, i, k, l} H_{a, \alpha,(k+1) b+j}(x),
$$

where $H_{a, \alpha,(k+1) b+j}(x)$ is the $c d f$ of the EL with parameters $a, \alpha$ and $(k+1) b+j$.

\section{Statistical Properties}

Established algebraic expansions to determine some structural properties of the TWL distribution can be more efficient than computing those directly by numerical integration of its density function. The statistical properties of the TWL distribution including quantile and random number generation, moments, factorial moments, cumulants, moment generating function, incomplete moments, mean deviations, probability weighted moments and Rényi and q entropies are discussed in this section. 


\subsection{Quantile and Random Number Generation}

The quantile function ( $q f$ ) of $X$, where $X: \operatorname{TWL}(\alpha, \beta, \lambda, a, b)$, is obtained by inverting (4) as

$$
x_{q}=\beta\left\{\left[\left(\ln (1-D)^{-1 / a}\right)^{1 / b}+1\right]^{1 / \alpha}-1\right\}, 0 \leq q \geq 1,
$$

where $D=\frac{1}{2 \lambda}\left\{(1+\lambda)-\sqrt{(1+\lambda)^{2}-4 \lambda q}\right\}$ and $\lim _{\lambda \rightarrow 0} D=q$.

By putting $q=0.5$ in Equation (11) we can get the median of $X$.

Simulating the TWL random variable is straightforward. If $U$ is a uniform variate on the unit interval $(0,1)$, then the random variable $X=x_{q}$ follows (5), i.e. $X: \operatorname{TWL}(\alpha, \beta, \lambda, a, b)$.

\subsection{Moments}

The $r$ th moment, denoted by $\mu_{r}$, of the $\operatorname{TWL}(\alpha, \beta, \lambda, a, b, x)$ is given by the following theorem.

Theorem 1. If $X$ is a continuous random variable has the $\operatorname{TWL}(\alpha, \beta, \lambda, a, b, x)$, then the $r$ th non-central moment of $X$, is given as follows

$$
\mu_{r}=\sum_{j, i, k, l=0}^{\infty} u_{j, i, k, l} \int_{0}^{\infty} x^{r} g_{a, \alpha,(k+1) b+j}(x) d x .
$$

using (7) we obtain (for $r \leq \alpha$ )

$$
\mu_{r}^{\prime}=\beta^{r} \sum_{m=0}^{r} \sum_{j, i, k, l=0}^{\infty}(-1)^{m}[(k+1) b+j]\left(\begin{array}{c}
r \\
m
\end{array}\right) u_{j, i, k, l} B\left\{[(k+1) b+j], \frac{m-r}{\alpha}+1\right\}
$$

Setting $r=1$ in (13), we have the mean of $X$. Then we can get the variance by the relation $\operatorname{Var}(X)=E\left(X^{2}\right)-E^{2}(X)$.

Based on the above Theorem (1) the coefficient of variation, coefficient of skewness and coefficient of kurtosis of the TWL $(\alpha, \beta, \lambda, a, b, x)$ distribution can be obtained according to the well-known relations.

Corollary 2. Using the relation between the central moments and non-centeral moments, we can obtain the $n$th central moment, denoted by $M_{n}$, of a TWL random variable as follows

$$
M_{n}=E(X-\mu)^{n}=\sum_{r=0}^{n}\left(\begin{array}{l}
n \\
r
\end{array}\right)(-\mu)^{n-r} E\left(X^{r}\right)
$$


then,

$$
M_{n}=\sum_{r=0}^{n}\left(\begin{array}{l}
n \\
r
\end{array}\right)(-1)^{n-r}\left(\mu_{1}\right)^{n-r} \mu_{r}
$$

and cumulants $\left(\kappa_{n}\right)$ of $X$ are obtained from (13) as

$$
\kappa_{n}=\mu_{n}^{\prime}-\sum_{r=0}^{n-1}\left(\begin{array}{c}
n-1 \\
r-1
\end{array}\right) \kappa_{r} \mu_{n-r}^{\prime},
$$

where $\kappa_{1}=\mu_{1}^{\prime}$ thus $\kappa_{2}=\mu_{2}^{\prime}-\mu_{1}^{2}, \kappa_{3}=\mu_{3}^{\prime}-3 \mu_{2}^{\prime} \mu_{1}^{\prime}+\mu_{1}^{\prime}$ etc. The skewness and kurtosis measures can be calculated from the ordinary moments using well-known relationships.

The $n$th descending factorial moment of $X$ (for $n=1,2, \ldots$ ) is

$$
\mu_{[1]}^{\prime}=E\left(X^{[1]}\right)=E[X(X-1) \times \ldots \times(X-n+1)]=\sum_{j=0}^{n} s(n, j) \mu_{j}^{\prime},
$$

where

$$
s(n, j)=\frac{1}{j !}\left[\frac{d^{j}}{d x^{j}}\left(j^{(n)}\right)\right]_{x=0} .
$$

is the Stirling number of the first kind.

\subsection{Moment Generating Function}

The moment generating function $(m g f)$ of the TWL distribution is given by the following theorem.

Theorem 2. If $X$ is a continuous random variable has the $\operatorname{TWL}(\alpha, \beta, \lambda, a, b, x)$, then the moment generating function (mgf) of $X$, denoted by $M_{X}(t)=E\left(e^{t X}\right)$, is given as follows

$$
\begin{aligned}
& M_{X}(t)=\alpha \sum_{j, i, k, l, m, r=0}^{\infty} \frac{(-1)^{m} r ![(k+1) b+j]}{\beta^{r+1}} \\
& \times\left(\begin{array}{c}
{[(k+1) b+j]-1} \\
m
\end{array}\right)\left(\begin{array}{c}
-(m+1) \alpha-1 \\
r
\end{array}\right) u_{j, i, k, l}(-t)^{-(r+1)} .
\end{aligned}
$$

\subsection{Incomplete Moments}

The important application of the first incomplete moment refers to the Bonferroni and Lorenz curves. These curves are very useful in economics, reliability, demography, insurance and medicine. The answers to many important questions in economics require more than just knowing the mean of the distribution, but its shape as well. This is obvious not only in the study of econometrics but in other areas as well. The $s^{\text {th }}$ incomplete moments, denoted by $\varphi_{s}(t)$, of the TWL r.v. is given by

$$
\varphi_{s}(t)=\int_{0}^{t} x^{s} f(x) d x
$$


Using Equation (8) and the lower incomplete gamma function, we obtain(for $s \leq \alpha$ )

$$
\varphi_{s}(t)=\beta^{s} \sum_{m=0}^{r} \sum_{j, i, k, l=0}^{\infty}(-1)^{m}[(k+1) b+j]\left(\begin{array}{c}
s \\
m
\end{array}\right) u_{j, i, k, l} B\left\{[(k+1) b+j], \frac{m-s}{\alpha}+1\right\}
$$

Another application of the first incomplete moment is related to the mean residual life and the mean waiting time given by $m_{1}(t ; v)=\left(1-\varphi_{1}(t)\right) / R(t ; v)-t$ and $M_{1}(t ; v)=t-\left(\varphi_{1}(t) / F(t ; v)\right)$, respectively.

Furthermore, the amount of scatter in a population is evidently measured to some extent by the totality of deviations from the mean and median. The mean deviations about the mean $\left(\delta_{\mu}(X)=E\left(\left|X-\mu_{1}\right|\right)\right)$ and about the median $\left(\delta_{\mu}(X)=E(|X-M|)\right)$ of $X$ can be, used as measures of spread in a population, expressed by

$$
\delta_{\mu}(X)=\int_{0}^{\infty}\left|X-\mu_{1}^{\prime}\right| f(x) d x=2 \mu_{1}^{\prime} F\left(\mu_{1}^{\prime}\right)-2 \varphi_{1}\left(\mu_{1}^{\prime}\right),
$$

and

$$
\delta_{M}(X)=\int_{0}^{\infty}|X-M| f(x) d x=\mu_{1}^{\prime}-2 \varphi_{1}(M),
$$

respectively, where $\mu_{1}^{\prime}=E(X)$ comes from (13), $F\left(\mu_{1}^{\prime}\right)$ is simply calculated from (4), $\varphi_{1}\left(\mu_{1}^{\prime}\right)$ is the first incomplete moments and $M$ is the median of $X$.

\subsection{Probability weighted moments}

The probability weighted moments (PWMs) are used to derive estimators of the parameters and quantiles of generalized distributions. These moments have low variance and no severe bias, and they compare favorably with estimators obtained by the maximum likelihood method The $(s ; r)$ th PWM of $X$

( for $r \geq 1, s \geq 0$ ) is formally defined by

$$
\rho_{r, s}=E\left[X^{r}(F(x))^{s}\right]=\int_{0}^{\infty} x^{r}(F(x))^{s} f(x) d x
$$

We can write from (3)

$$
\begin{aligned}
& F^{s}(x, a, b, \lambda, \alpha, \beta)=\sum_{w, h=0}^{\infty} \underbrace{(-1)^{w+h}\left(\begin{array}{c}
s \\
w
\end{array}\right)\left(\begin{array}{c}
w+s \\
h
\end{array}\right)(1+\lambda)^{s-w} \lambda^{w}}_{v_{w, h}} \\
& \quad \times \exp \left\{-a h\left\{\left[\left(1+\frac{x}{\beta}\right)^{\alpha}-1\right]\right\}\right\} .
\end{aligned}
$$


Therefore, from Equations (3) and (4) we can express $\rho_{r, s}$ as

$$
\rho_{r, s}=\sum_{w, h=0}^{\infty} \frac{v_{w, h}}{(h+1)} \int_{0}^{\infty} x^{r} f(x, \alpha, \beta, \lambda, a(h+1), b) d x .
$$

By using (13) we obtain (for $r \leq \alpha$ )

$$
\rho_{r, s}=\beta^{r} \sum_{j, i, k, l, h, w=0}^{\infty} \frac{(-1)^{w+h}}{(h+1)}\left(\begin{array}{c}
s \\
w
\end{array}\right)\left(\begin{array}{c}
w+s \\
h
\end{array}\right) s_{j: w} \sum_{m=0}^{r} \mathrm{~B}\left([(k+1) b+j], \frac{m-r}{\alpha}+1\right),
$$

where

$$
\begin{aligned}
s_{j: w} & =\frac{(-1)^{j+i+k+l} 2^{i} a^{k} \lambda^{w+1}(1+\lambda)^{s-w+i-1}}{j ! k !} \\
& \times\left(\begin{array}{l}
i \\
l
\end{array}\right)\left(\begin{array}{l}
1 \\
i
\end{array}\right) \frac{\Gamma[(k+1) b+j+1][(l+1)(h+1)]^{k}}{\Gamma[(k+1) b+1][(k+1) b+j]} .
\end{aligned}
$$

\subsection{Rényi and q-Entropies}

The Rényi entropy of a random variable $X$ represents a measure of variation of the uncertainty. The Rényi entropy is defined by

$$
I_{\theta}(X)=\frac{1}{1-\theta} \log \int_{-\infty}^{\infty} f^{\theta}(x) d x, \theta>0 \text { and } \theta \neq 1 .
$$

Therefore, the Rényi entropy of a random variable $X$ which follows the TWL is given by

where

$$
I_{\theta}(X)=\frac{1}{1-\theta} \log \left[(b \alpha)^{\theta} \sum_{j, i, k, l=0}^{\infty} \varsigma_{j, i, k, l}\right]
$$

$$
\begin{aligned}
S_{j, i, k, l} & =a^{k+\theta} \beta^{m-\theta+1} \frac{(-1)^{i+k+l}(2 \lambda)^{i}(1+\lambda)^{\theta-i} \Gamma[\theta(b+1)+b k+j]}{k ! m ! \Gamma[\theta(b+1)+b k]} \\
\times & \frac{\Gamma[m-\theta(b-1)+b k+j](l+\theta)^{k}}{\Gamma[b k-\theta(b+1)+j][m \alpha+\theta(\alpha+1)-1]}\left(\begin{array}{l}
\theta \\
i
\end{array}\right)\left(\begin{array}{l}
i \\
l
\end{array}\right) .
\end{aligned}
$$

The q-entropy, say $H_{q}(X)$, is defined by

$$
\begin{aligned}
& H_{q}(X)=\frac{1}{q-1} \log \left\{1-\int_{-\infty}^{\infty} f^{q}(x) d x\right\}, q>0 \text { and } q \neq 1 . \\
& H_{q}(X)=\frac{1}{q-1} \log \left\{1-\left[(b \alpha)^{q} \sum_{j, i, k, l=0}^{\infty} d_{j, i, k, l}\right]\right\},
\end{aligned}
$$


where

$$
\begin{aligned}
d_{j, i, k, l} & =a^{k+q} \beta^{m-q+1} \frac{(-1)^{i+k+l}(2 \lambda)^{i}(1+\lambda)^{q-i} \Gamma[q(b+1)+b k+j]}{k ! m ! \Gamma[q(b+1)+b k]} \\
\times & \frac{\Gamma[m-q(b-1)+b k+j](l+q)^{k}}{\Gamma[b k-q(b+1)+j][m \alpha+q(\alpha+1)-1]}\left(\begin{array}{l}
q \\
i
\end{array}\right)\left(\begin{array}{l}
i \\
l
\end{array}\right) .
\end{aligned}
$$

\section{Order Statistics}

If $X_{1}, X_{2}, \ldots, X_{n}$ is a random sample of size $n$ from the TWL distribution and $X_{(1)}, X_{(2)}, \ldots, X_{(n)}$ be the corresponding order statistics. Then the $p d f$ of $\mathrm{jth}$ order statistics denoted by $X_{i: n}, f_{i: n}(x)$ is given by

$$
f_{i: n}(x)=\frac{f(x)}{\mathrm{B}(i, n-i+1)} \sum_{j=0}^{n-i}(-1)^{j}\left(\begin{array}{c}
n-1 \\
j
\end{array}\right) F^{i+j-1}(x)
$$

Therefore, we can write

$$
F^{j+i-1}(x)=\sum_{m=0}^{\infty}(-1)^{m}\left(\begin{array}{c}
j+l+i-1 \\
m
\end{array}\right) \exp \left\{-a m\left\{\left(1+\frac{x}{\beta}\right)^{\alpha}-1\right\}^{b}\right\}
$$

and then by inserting (4) in equation (19), we obtain

$$
f_{i: n}(x)=\sum_{m=0}^{\infty} b_{m+1} f(x, \alpha, \beta, \lambda, a(m+1), b),
$$

where

$$
\begin{aligned}
& b_{m+1}=\frac{\ell}{(m+1) \mathrm{B}(i, n-i+1)} \sum_{j=0}^{n-i}(-1)^{j+m}\left(\begin{array}{c}
n-i \\
j
\end{array}\right)\left(\begin{array}{c}
j+i-1 \\
m
\end{array}\right) . \\
& =\sum_{l=0}^{\infty}(-1)^{l}(1+\lambda)^{j+i-l-1} \lambda^{l}\left(\begin{array}{c}
j+i-1 \\
l
\end{array}\right) .
\end{aligned}
$$

and $f(x, \alpha, \beta, \lambda, a(m+1), b)$ denotes the TWL density function with parameters $\alpha, \beta, \gamma, a(m+1)$ and $b$. So the density function of the TWL order statistics is a mixture of WL densities. Based on equation (20), we can obtain some structural properties of $X_{i: n}$ from those TWL properties .

The $r$ th moment of $X_{i: n}$ (for $r<\alpha$ ) follows from (14) and (20) as

$$
E\left(X_{i: n}^{r}\right)=\beta^{r} \sum_{m=0}^{r}(-1)^{m}\left(\begin{array}{c}
r \\
m
\end{array}\right) b_{m+1}+\sum_{j, k, l=0}^{\infty}[(k+1) b+j] u_{j, i, k, l} \mathrm{~B}\left([(k+1) b+j], \frac{m-r}{\alpha}+1\right) .
$$

The L-moments are analogous to the ordinary moments but can be estimated by linear combinations of order statistics. They exist whenever the mean of the distribution exists, even though some higher moments may not exist, and are relatively robust to the effects 
of outliers. Based upon the moments in Equation (21), we can derive explicit expressions for the L-moments of $X$ as infinite weighted linear combinations of the means of suitable TWL distributions. They are linear functions of expected order statistics defined by

$$
\lambda_{r}=\frac{1}{r} \sum_{d=0}^{r-1}(-1)^{d}\left(\begin{array}{c}
r-1 \\
d
\end{array}\right) E\left(X_{r-d: d}\right), r \geq 1
$$

The first four L-moments are given by $\lambda_{1}=E\left(X_{1: 1}\right), \lambda_{2}=\frac{1}{2} E\left(X_{2: 2}-X_{1: 2}\right), \lambda_{3}=\frac{1}{3} E\left(X_{3: 3}-2 X_{2: 3}+X_{1: 3}\right)$ and $\lambda_{4}=\frac{1}{4} E\left(X_{4: 4}-3 X_{3: 4}+3 X_{2: 4}-X_{1: 4}\right)$.

One simply can obtain the $\lambda$ 's for $X$ from Equation (21) with $q=1$.

\section{Estimation}

The maximum likelihood estimators (MLEs) for the parameters of the TWL $(\alpha, \beta, \lambda, a, b, x)$ is discussed in this section. Let $\mathbf{X}=\left(X_{1}, \ldots, X_{n}\right)$ be a random sample of this distribution with unknown parameter vector $v=(\alpha, \beta, \gamma, a, b)^{T}$. The likelihood function for $v, l(v ; x)$ is

$$
\begin{aligned}
& l(v ; x)=\left(\frac{a b \alpha \gamma}{\beta}\right)^{n} \prod_{i=1}^{n}\left(1+\frac{x_{i}}{\beta}\right)^{b \alpha-1} \prod_{i=1}^{n}\left[1-\left(1+\frac{x_{i}}{\beta}\right)^{-\alpha}\right]^{b-1} \\
& \prod_{i=1}^{n} \exp \left\{-a\left\{\left(1+\frac{x_{i}}{\beta}\right)^{\alpha}-1\right\}\right\} \\
& \prod_{i=1}^{n}\left[(1+\lambda)-2 \lambda\left(1-\exp \left\{-a\left\{\left(1+\frac{x_{i}}{\beta}\right)^{\alpha}-1\right\}\right\}\right) .\right.
\end{aligned}
$$

Then, the log-likelihood function, $\ell$, becomes:

$$
\begin{aligned}
\ell= & n(\ln a+\ln b+\ln \alpha+\ln \lambda-\ln \beta)+(\alpha b-1)_{i=1}^{n} \ln \left(1+\frac{x_{i}}{\beta}\right) \\
& +(b-1) \sum_{i=1}^{n} \ln \left[1-\left(1+\frac{x_{i}}{\beta}\right)^{-\alpha}\right]-a_{i=1}^{n} \ln Z_{i}^{b}+{ }_{i=1}^{n} \ln Q_{i},
\end{aligned}
$$

where $Z_{i}=S_{i}^{\alpha}-1, S_{i}=1+\frac{x}{\beta}, Q_{i}=\left\{(1+\lambda)-2 \lambda\left[1-\exp \left(-a Z_{i}^{b}\right)\right]\right\}$

Therefore the score vector is $\mathbf{U}(v)=\frac{\partial \ell}{\partial v}=\left(\frac{\partial \ell}{\partial \alpha}, \frac{\partial \ell}{\partial \beta}, \frac{\partial \ell}{\partial \lambda}, \frac{\partial \ell}{\partial a}, \frac{\partial \ell}{\partial b}\right)^{T}$ 
Let $P_{i}=1-e^{-a z_{i}^{b}}$.

$$
\begin{aligned}
& \frac{\partial \ell}{\partial \alpha}=\frac{n}{\alpha}+b \sum_{i=1}^{n} \ln \left(S_{i}\right)+(b-1) \sum_{i=1}^{n} \frac{S_{i}^{-\alpha} \ln \left(S_{i}\right)}{1-S_{i}^{-\alpha}} \\
& -a b \sum_{i=1}^{n} Z_{i}^{b-1} S_{i} \alpha \ln S_{i}-2 a b \lambda \sum_{i=1}^{n} \frac{Z_{i}^{b} S_{i}^{\alpha} e^{-a Z_{i}^{b}} \ln \left(S_{i}\right)}{Q_{i}}, \\
& \frac{\partial \ell}{\partial \beta}=-\frac{n}{\beta}-\frac{(b \alpha-1)}{\beta^{2}} \sum_{i=1}^{n} x_{i} S_{i}^{-1}+\frac{\alpha(b-1)}{\beta^{2}} \sum_{i=1}^{n} \frac{x_{i} S_{i}^{-\alpha-1}}{1-S_{i}^{-\alpha}} \\
& \quad+\frac{a b \alpha}{\beta^{2} \sum_{i=1}^{n} x_{i} Z_{i}^{b-1} S^{\alpha-1}-\frac{2 a b \alpha \lambda}{\beta^{2}} \sum_{i=1}^{n} \frac{x S_{i}^{\alpha-1} Z_{i}^{b-1} e^{-a Z_{i}^{b}}}{Q_{i}}} \\
& \frac{\partial \ell}{\partial \lambda}=\sum_{i=1}^{n} \frac{1-2\left(1-e^{-a Z_{i}^{b}}\right)}{Q_{i}} \\
& \frac{\partial \ell}{\partial a}=\frac{n}{a}-\sum_{i=1}^{n} Z_{i}^{b}-2 \lambda \sum_{i=1}^{n} \frac{Z_{i}^{b} e^{-a Z_{i}^{b}}}{Q_{i}}
\end{aligned}
$$

and

$$
\begin{aligned}
\frac{\partial \ell}{\partial a}=\frac{n}{b} & +\alpha \sum_{i=1}^{n} \ln \left(S_{i}\right)-\sum_{i=1}^{n} \ln \left(1-S_{i}^{-\alpha}\right)-a \sum_{i=1}^{n} Z_{i}^{b} \ln \left(Z_{i}\right) \\
& -2 a \lambda \sum_{i=1}^{n} \frac{Z_{i}^{b} e^{-a Z_{i}^{b}} \ln \left(Z_{i}\right)}{Q_{i}} .
\end{aligned}
$$

We can find the estimates of the unknown parameters by setting the score vector to zero, $\mathbf{U}(v)=0$, and solving them simultaneously yields the ML estimators $\hat{\alpha}, \hat{\beta}, \hat{\lambda}, \hat{a}$ and $\hat{b}$. These equations cannot be solved analytically and statistical software can be used to solve them numerically by means of iterative techniques such as the Newton-Raphson algorithm. For the five parameters TWL distribution all the second order derivatives exist.

For interval estimation of the model parameters, we require the $5 \times 5$ observed information matrix $J(v)=\left\{J_{r s}\right\}($ for $r, s=\alpha, \beta, \lambda, a, b)$. Under standard regularity conditions, the multivariate normal $N_{5}\left(0, J(v)^{-1}\right)$ distribution can be used to construct approximate confidence intervals for the model parameters. Here, $J(v)$ is the total observed information matrix evaluated at $v$. Therefore, Approximate $100(1-\phi) \%$ confidence intervals for $\alpha, \beta, \lambda, a$ and $b$ can be determined as:

$\hat{\alpha} \pm Z_{\frac{\phi}{2}} \sqrt{J_{\alpha \alpha}}, \quad \hat{\beta} \pm Z_{\frac{\phi}{2}} \sqrt{J_{\beta \beta}}, \quad \hat{\lambda} \pm Z_{\frac{\phi}{2}} \sqrt{J_{\lambda \lambda}}, \quad \hat{a} \pm Z_{\frac{\phi}{2}} \sqrt{J_{a a}}$ and $\hat{b} \pm Z_{\frac{\phi}{2}} \sqrt{J_{b b}}$, where $Z_{\frac{\phi}{2}}$ is the upper $\phi$ th percentile of the standard normal distribution. 


\section{Data Analysis}

In this section, we provide an application of the proposed TWL distribution to show the importance of the new model, where the TWL model is compared with other related models, namely Weibull Lomax (WL), McDonald Lomax (McL), transmuted complementary Weibull geometric (TCWG), modified beta Weibull (MBW) and Lomax (L) distributions.

The $p d f$ s of these, non-nested, models are given as follow:

- The transmuted complementary Weibull geometric (TCWG) distribution introduced by Afify et al. (2014). The pdf of TCWG distribution is

$$
f(x, \alpha, \beta, \lambda, a)=\frac{\alpha \beta a(a x)^{\beta-1} e^{-(a y)^{\beta}}\left[\alpha(1-\lambda)-(\alpha-\alpha \lambda-\lambda-1) e^{-(a x)^{\beta}}\right]}{\left(\alpha+(1-\alpha) e^{-(a x)^{\beta}}\right)^{3}},
$$

(where $x>0, \alpha, \beta, a>0,|\lambda| \leq 1)$.

- $\quad$ The modified beta Weibull (MBW) distribution introduced by Khan (2015). The pdf of MBW distribution is

$$
\begin{gathered}
f(y, \alpha, \beta, \lambda, a, b)=\frac{\beta \lambda^{a}}{\alpha^{\beta} B(a, b)} x^{\beta-1} e^{-b\left(\frac{x}{\alpha}\right)^{\beta}}\left[1-e^{-\left(\frac{x}{\alpha}\right)^{\beta}}\right]^{a-1} \\
\left\{1-(1-\lambda)\left[1-e^{-\left(\frac{x}{\alpha}\right)^{\beta}}\right]\right\}^{-(a+b)},
\end{gathered}
$$

(where $x>0, \alpha, \beta, \lambda, a, b>0$ ).

- $\quad$ The McDonald-Lomax (McL) distribution introduced by Lemonte and Cordeiro (2013). The pdf of McL distribution is

$$
\begin{aligned}
& f(x, \alpha, \beta, \lambda, a, b)=\frac{\alpha \lambda}{\beta B\left(a \lambda^{-1}, b\right)}\left(1+\frac{x}{\beta}\right)^{-(\alpha+1)}\left[1-\left(1+\frac{x}{\beta}\right)^{-\alpha}\right]^{a-1} \\
& \quad \times\left\{1-\left[1-\left(1+\frac{x}{\beta}\right)^{-\alpha}\right]^{\lambda} .\right.
\end{aligned}
$$

(where $x>0, \alpha, \beta, \lambda, a, b>0$ ).

The data set (gauge lengths of $10 \mathrm{~mm}$ ) from Kundu and Raqab (2009). This data set consists of, 63 observations: 1.901, 2.132, 2.203, 2.228, 2.257, 2.350, 2.361, 2.396, $2.397,2.445,2.454,2.474,2.518,2.522,2.525,2.532,2.575,2.614,2.616,2.618,2.624$, $2.659,2.675,2.738,2.740,2.856,2.917,2.928,2.937,2.937,2.977,2.996,3.030,3.125$, $3.139,3.145,3.220,3.223,3.235,3.243,3.264$, 3.272, 3.294, 3.332, 3.346, 3.377, 3.408, $3.435,3.493,3.501,3.537,3.554,3.562,3.628$, 3.852, 3.871, 3.886, 3.971, 4.024, 4.027, $4.225,4.395,5.020$. This data set is previously studied by Afify et al. (2015) to fit the exponentiated transmuted generalized Rayleigh (ETGR) distribution. 
In order to compare the distributions, we consider some criteria like $-2 \ell($. (Log-likelihood), AIC (Akaike Information Criterion) and CAIC (the consistent Akaike Information Criterion). furthermore, We also consider the Cram ér--von Mises $\left(W^{*}\right)$ and Anderson--Darling $\left(A^{*}\right)$ statistics. The statistics $W^{*}$ and $A^{*}$ are described in details in Chen and Balakrishnan (1995). In general, the smaller the values of these statistics ( $A I C, C A I C, W^{*}$ and $A^{*}$ ), the better the fit to the data, where

$$
\begin{aligned}
& A I C=-2 \ell+2 k, C A I C=-2 \ell+2 k n /(n-k-1), \\
& W^{*}=\left(\frac{1}{2 n}+1\right)\left[{ }_{j=1}^{n}\left(z_{i}-\frac{2 j-1}{2 n}\right)^{2}+\frac{1}{12 n}\right],
\end{aligned}
$$

and

$$
A^{*}=\left(\frac{9}{4 n^{2}}+\frac{3}{4 n}+1\right)\left[n+\frac{1}{n}_{j=1}^{n}(2 j-1) \log \left[z_{i}\left(1-z_{n-j+1}\right)\right]\right] .
$$

where $\ell$ denotes the log-likelihood function evaluated at the maximum likelihood estimates, $k$ is the number of parameters, $n$ is the sample size and $z_{i}=F\left(y_{j}\right)$ where the $y_{j}$ values being the ordered observations.

Table 1 lists the numerical values of the $-2 \ell(),. A I C, C A I C, W^{*}$ and $A^{*}$, whilst the MLEs and their corresponding standard errors (in parentheses) of the model parameters are shown in tables 2, respectively. These numerical results are obtained using the MATH- CAD PROGRAM.

Table 1: The statistics $-2 \ell(),. A I C, C A I C, W^{*}$ and $A^{*}$ for gauge lengths of $10 \mathrm{~mm}$ data set

\begin{tabular}{cccccc}
\hline \hline Model & \multicolumn{5}{c}{ Goodness of fit criteria } \\
\hline & $-2 \ell()$. & $A I C$ & $C A I C$ & $W^{*}$ & $A^{*}$ \\
\hline TWL & $\mathbf{1 1 9 . 6 8 8}$ & $\mathbf{1 2 9 . 6 8 8}$ & 130.741 & $\mathbf{0 . 1 0 7 1 9}$ & $\mathbf{0 . 7 3 0 7 2}$ \\
\hline WL & 121.787 & 129.787 & $\mathbf{1 3 0 . 4 7 6}$ & 0.1174 & 0.81217 \\
\hline MBW & 125.917 & 135.917 & 136.97 & 0.15171 & 1.04361 \\
\hline TCWG & 126.895 & 134.895 & 135.585 & 0.17139 & 1.17435 \\
\hline McL & 130.597 & 140.597 & 141.65 & 0.10814 & 0.81415 \\
\hline L & 266.921 & 270.921 & 271.121 & 0.50412 & 7.6338 \\
\hline \hline
\end{tabular}


Table 2: MLEs and their standard errors (in parentheses) for gauge lengths of 10 mm data set

\begin{tabular}{cccccc}
\hline \hline Model & \multicolumn{5}{c}{ Estimates } \\
\hline & $\hat{\alpha}$ & $\hat{\beta}$ & $\lambda$ & $a$ & $\hat{b}$ \\
\hline TWL & 0.3922 & 0.6603 & 0.7364 & 0.5287 & 8.4451 \\
& $(0.339)$ & $(1.174)$ & $(0.286)$ & $(3.32)$ & $(4.397)$ \\
\hline WL & 0.2471 & 0.3255 & -- & 8.003 & 10.1306 \\
& $(0.06)$ & $(0.571)$ & -- & $(49.403)$ & $(4.602)$ \\
\hline MBW & 1.8974 & 5.2354 & 17.2067 & 6.4617 & 0.0514 \\
& $(0.283)$ & $(0.435)$ & $(13.115)$ & $(2.277)$ & $(0.034)$ \\
\hline TCWG & 0.2022 & 3.3482 & -0.0001 & 0.3876 & -- \\
& $(0.217)$ & $(0.783)$ & $(0.496)$ & $(0.069)$ & -- \\
\hline McL & 45.9249 & 48.3024 & 353.1435 & 18.1192 & 195.4633 \\
& $(59.312)$ & $(63.047)$ & $(375.678)$ & $(8.855)$ & $(123.217)$ \\
\hline L & 2545.5181 & 7786.421 & -- & -- & -- \\
& $(16520)$ & $(50540)$ & -- & -- & - \\
\hline \hline
\end{tabular}

Table 1 compares the TWL model with the WL, McL, TCWG, MBW, and Lomax models. We note that the TWL model gives the lowest values for the $A I C, C A I C, W^{*}$ and $A^{*}$ statistics (except $C A I C$ ) among all fitted models. So, the TWL model could be chosen as the best model.

\section{Conclusions}

In this paper, We propose a new five-parameter model, called the transmuted Weibull Lomax (TWL) distribution, which extends the Weibull Lomax (WL) distribution introduced by Tahir et al. (2015). An obvious reason for generalizing a standard distribution is the fact that the generalization provides more flexibility to analyze real life data. We provide some of its mathematical and statistical properties. The TWL density function can be expressed as a mixture of exponentiated Lomax (EL) densities. We derive explicit expressions for the ordinary and incomplete moments, factorial moments, cumulants, generating function, probability weighted moments, Rényi and q-entropies. We also obtain the density function of the order statistics and its moments. We discuss maximum likelihood estimation. The proposed distribution was applied to a real data set; it was shown to provide a better fit than several other related models and non-nested models. We hope that the proposed model will attract wider application in areas such as engineering, survival and lifetime data, meteorology, hydrology, economics (income inequality) and others. 


\section{References}

1. Abdul-Moniem, I. B. and Abdel-Hameed, H. F. (2012). On exponentiated Lomax distribution, International Journal of Mathematical Archive 3, 2144-2150.

2. Afify, A. Z., Nofal, Z. M. and Butt, N. S. (2014). Transmuted Complementary Weibull Geometric Distribution. Pak.j.stat.oper.res. Vol.X, No. 4. 435-454.

3. Arnold, B.C. (1983). Pareto Distributions. International Cooperative Publishing House, Maryland.

4. Balkema, A.A. and de Hann, L. (1974). Residual life at great age, Annals of Probability 2, 972-804.

5. Ashour, S. K. and Eltehiwy, M. A. (2013). Transmuted Lomax Distribution. American Journal of Applied Mathematics and Statistics, Vol. 1, No. 6, 121-127.

6. Chahkandi, M. and Ganjali, M. (2009). On some lifetime distributions with decreasing failure rate, Computational Statistics and Data Analysis 53, 4433-4440.

7. Chen, G., Balakrishnan, N. (1995). A general purpose approximate goodness-offit test. Journal of Quality Technology 27, 154-161.

8. Cordeiro, G. M., Ortega, E. M. M. and Popovic, B. V. (2013). The gamma-Lomax distribution, Journal of Statistical computation and Simulation iFirst, doi:10.1080/00949655. 822869.

9. Ghitany, M. E., AL-Awadhi, F. A and Alkhalfan, L. A. (2007). Marshall-Olkin extended Lomax distribution and its applications to censored data, Communications in Statistics--Theory and Methods 36, 1855-1866.

10. Johnson, N. L., Kotz, S. and Balakrishnan, N. (1994). Contineous Univariate Distributions: Vol. 1, 2nd edition Wiley, New York.

11. Khan, M. N. (2015). The modified beta Weibull distribution with applications. To appear in the Hacettepe Journal of Mathematics and Statistics.

12. Kundu, D. and Raqab, M. Z. (2009). Estimation of $\mathrm{R}=\mathrm{P}(\mathrm{Y}<\mathrm{X}$ ) for threeparameter Weibull distribution. Statistics and Probability Letters. Vol. 79, 1839-1846.

13. Lemonte, A. J. and Cordeiro, G. M. (2013). An extended Lomax distribution, Statistics 47, 800-816.

14. Lomax, K. S. (1954). Business failures. Another example of the analysis of failure data, J. Amer. Statist. Assoc. 49, 847-852.

15. Shaw, W. T. and Buckley, I. R. C. (2007). The alchemy of probability distributions: beyond Gram-Charlier expansions and a skew-kurtotic-normal distribution from a rank transmutation map. Research report.

16. Tahir, M. H., Cordeiro, G. M., Mansoor, M. and Zubair, M. (2015). The WeibullLomax distribution: properties and applications. To appear in the Hacettepe Journal of Mathematics and Statistics. 